\title{
Browning of Epicatechin (EC) and Epigallocatechin (EGC) by Auto- Oxidation
}

\author{
Junfeng Tan, Wouter J. C. de Bruijn, Annemiek van Zadelhoff, Zhi Lin,* and Jean-Paul Vincken* \\ Cite This: J. Agric. Food Chem. 2020, 68, 13879-13887 \\ Read Online
}

ABSTRACT: Green tea catechins are well known for their health benefits. However, these compounds can easily be oxidized, resulting in brown color formation, even in the absence of active oxidative enzymes. Browning of catechin-rich beverages, such as green tea, during their shelf life is undesired. The mechanisms of auto-oxidation of catechins and the brown products formed are still largely unknown. Therefore, we studied auto-oxidative browning of epicatechin (EC) and epigallocatechin (EGC) in model systems. Products of EC and EGC auto-oxidation were analyzed by reversed-phase ultra-high-performance liquid chromatography with photodiode array detection coupled to mass spectrometry (RP-UHPLC-PDA-MS). In the EC model system, $11 \delta$-type dehydrodicatechins $\left(\mathrm{DhC}_{2} \mathrm{~s}\right)$ and $18 \delta$-type dehydrotricatechins $\left(\mathrm{DhC}_{3} \mathrm{~s}\right)$ that were related to browning could be tentatively identified by their $\mathrm{MS}^{2}$ signature fragments. In the EGC model system, auto-oxidation led to the formation of 13 dihydro-indenecarboxylic acid derivatives and 2 theaflagallins that were related to browning. Based on the products formed, we propose mechanisms for the auto-oxidative browning of EC and EGC. Furthermore, our results indicate that dimers and oligomers that possess a combination of an extended conjugated system, fused rings, and carbonyl groups are responsible for the brown color formation in the absence of oxidative enzymes.

KEYWORDS: tea, catechins, nonenzymatic, oxidation, discoloration

\section{INTRODUCTION}

Catechins are a group of phenolic compounds that are abundantly present in green tea leaves. These phenolics are flavan-3-ols that are characterized by their meta-5,7-dihydroxysubstituted A-ring and catechol or pyrogallol B-ring (Figure $1 A, B)$. Epicatechin (EC) and epigallocatechin (EGC), two of the main catechins found in green tea, are colorless bioactive compounds, which can easily be oxidized. ${ }^{1,2}$ During fermentation of green tea leaves to produce black tea, oxidation of catechins is catalyzed by the enzymes polyphenol oxidase (PPO) and peroxidase (POD). ${ }^{3}$ The mechanism of browning by these endogenous enzymes has been studied extensively. However, browning by auto-oxidation of catechins can take place even when the enzymes are inactivated or removed. ${ }^{4}$ For instance, browning of catechin-rich ready-to-drink (RTD) green tea beverages during their shelf life is undesired and regarded as a quality issue. ${ }^{5}$ To mask the off-color formation, manufacturers commonly use green bottles or nontransparent packaging. Important as it is, relatively little research has been devoted to auto-oxidation of catechins in aqueous systems and the compositional changes of green tea upon storage have not yet been investigated in detail.

On the other hand, the chemical structures of red or brown enzymatic oxidation products present in black tea are well understood. For example, theaflavin ${ }^{6}$ (TF) (Figure 1C), which possesses a benzotropolone moiety, has a bright red color, and theacitrin C (TCC) (Figure 1D), which possesses fused rings with three carbonyl moieties, has a yellow color. $^{7-9}$ The common characteristics of red and brown pigments in black tea include that these compounds are condensation products derived from catechins and that they possess a dense region of conjugated double bonds. Formation of these pigments is initiated by formation of catechin-derived $o$-quinones by PPO or POD, with a subsequent series of follow-up reactions. Major follow-up reactions include oligomerization and intramolecular rearrangement. Oligomerization of catechins results in a larger molecular size with different interflavanic linkages, as exemplified by TF and TCC. Intramolecular rearrangements commonly require a further oxidation step of the oligomer without elongation; e.g., theanaphthoquinone (TNQ) (Figure $1 \mathrm{E})$ is formed by oxidation of $\mathrm{TF}$ and subsequent ring contraction. $^{10}$

For nonenzymatic oxidation, i.e., auto-oxidation, it is still largely unknown how these reactions are initiated and via what mechanisms browning occurs. ${ }^{4,5,11,12}$ We decided to focus on auto-oxidative browning of EC and EGC as a first step toward gaining insight into the auto-oxidative browning of catechinrich products, like RTD green tea. The aims of this study were (i) to specifically analyze the auto-oxidative browning products formed in EC and EGC model systems and (ii) to understand the reaction mechanisms underlying the formation of brown products upon auto-oxidation. To analyze the brown products, pure EC or EGC were incubated in the absence of enzymes. A

Received: September 4, 2020

Revised: October 26, 2020

Accepted: October 29, 2020

Published: November 10, 2020 
A<smiles>Cc1c(O)cc(O)cc1O[C@@H](c1ccc(O)c(O)c1)[C@H](O)CC(F)(F)F</smiles><smiles>Cc1cc(O)c2c(c1O)OC(c1cc(O)c(=O)c3c(O)c(O)cc(C4Oc5cc(O)cc(O)c5CC4O)c3c1)C(O)C2</smiles>

TF<smiles>[B]c1cc(O)c2c(c1)O[C@H](c1cc(O)c(O)c(O)c1)[C@H](O)C2</smiles>

D

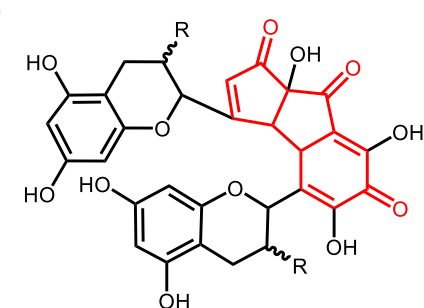

TCC<smiles>O=C1C=C(C2Oc3cc(O)cc(O)c3CC2O)c2cc(C3Oc4cc(O)cc(O)c4CC3O)cc(O)c2C1=O</smiles>

TNQ

Figure 1. Structures of EC and EGC and the key structural elements (in red) responsible for the brown color of theaflavin (TF), theacitrin C (TCC), and theanaphthoquinone (TNQ). R represents a galloyl ester.

chromatographic peak (UV-vis)-to-peak (full MS) matching strategy was used to determine the compounds that contribute to browning in EC and EGC model systems. The structure of these compounds was elucidated based on a combination of mass spectrometric methods, including IT-MS ${ }^{n}$ and FTMS $^{2}$.

\section{MATERIALS AND METHODS}

2.1. Materials. Sunphenon- 94 , containing approximately $94 \%(\mathrm{w} /$ $\mathrm{w})$ epigallocatechin gallate $(\mathrm{EGCg})$ and $5 \%(\mathrm{w} / \mathrm{w})$ epicatechin gallate $(\mathrm{ECg})$, was purchased from Taiyo (Schwelm, Germany). $o$-Phenylenediamine was purchased from Sigma-Aldrich (St. Louis, MO). UHPLC-MS grade acetonitrile (ACN), formic acid (FA) 99\% (v/v), ACN with $0.1 \%(\mathrm{v} / \mathrm{v}) \mathrm{FA}$, and water with $0.1 \%(\mathrm{v} / \mathrm{v}) \mathrm{FA}$ were obtained from Biosolve (Valkenswaard, The Netherlands). Water for other purposes than UHPLC was prepared using a Milli-Q water purification system (Millipore, Billerica, MA).

2.2. Methods. 2.2.1. Epigallocatechin and Epicatechin Preparation and Purification. Epigallocatechin (EGC) and epicatechin (EC) were prepared from Sunphenon-94 by treatment with tannase, as described elsewhere. ${ }^{13}$ EGC and EC were further purified on a Reveleris flash chromatography system (Grace, Columbia, MD) equipped with a Reveleris $C_{18}$ RP $80 \mathrm{~g}$ cartridge (particle size $40 \mu \mathrm{m}$ ) (Büchi, Flawil, Switzerland). The compounds were eluted with water (A) and ACN (B), both containing $1 \%(\mathrm{v} / \mathrm{v})$ formic acid, at room temperature at a flow rate of $60 \mathrm{~mL} / \mathrm{min}$. The elution program used was as follows: isocratic at $0 \% \mathrm{~B}$ for $8 \mathrm{~min}$, linear gradient to $22 \% \mathrm{~B}$ from 8 to $38 \mathrm{~min}$, linear gradient to $100 \% \mathrm{~B}$ from 38 to $41 \mathrm{~min}$, and isocratic at $100 \%$ B from 41 to $46 \mathrm{~min}$. Fractions were collected and evaporated to dryness by subsequent rotary evaporation at $40{ }^{\circ} \mathrm{C}$ and freeze-drying, after which the compounds were stored at $-20^{\circ} \mathrm{C}$. The purity of both obtained catechins was $99 \%(\mathrm{w} / \mathrm{w})$ based on the UV $278 \mathrm{~nm}$ peak area as determined by RP-UHPLC-PDA.

2.2.2. EC and EGC Aqueous Model System Incubation. To assess the brown color formation of pure EC and EGC under the influence of temperature, we prepared $1 \mathrm{mM}$ aqueous solutions of each compound. The following samples were used for the model incubation: $4 \mathrm{~mL}$ EC $(0.30 \mathrm{mg} / \mathrm{mL}$ water $)$ and $4 \mathrm{~mL}$ EGC $(0.30$ $\mathrm{mg} / \mathrm{mL}$ water) in closed $10 \mathrm{~mL}$ glass tubes. The samples were placed in the dark in a water bath at $80^{\circ} \mathrm{C}$. Six separate tubes were prepared per compound, and at time points $0,1,2,4,6$, and $24 \mathrm{~h}$, a tube of each pure compound was taken and centrifuged $(10000 \mathrm{~g}, 5 \mathrm{~min}, 20$ $\left.{ }^{\circ} \mathrm{C}\right)$. Subsequently, $3 \mathrm{~mL}$ of each sample was transferred to a quartz cuvette with a stopper (Hellma Analytics, Müllheim, Germany) for spectrophotometric analysis, and the remaining sample was stored at $-20{ }^{\circ} \mathrm{C}$ until further analysis by RP-UHPLC-PDA-MS.

2.2.3. Treatment with o-Phenylenediamine. To investigate whether $o$-quinones were formed during auto-oxidation, $200 \mu \mathrm{L}$ of the EC or EGC sample after incubation (time point $4 \mathrm{~h}$, see Section 2.2.2) was treated with a solution of $40 \mu \mathrm{L}$ of $o$-phenylenediamine (10 $\mathrm{mg} / \mathrm{mL}$ in ACN) at $80{ }^{\circ} \mathrm{C}$ for $30 \mathrm{~min}$. Subsequently, $200 \mu \mathrm{L}$ of the reaction mixture was centrifuged $\left(10000 \mathrm{~g}, 5 \mathrm{~min}, 20^{\circ} \mathrm{C}\right)$ prior to analysis by RP-UHPLC-PDA-MS.

2.2.4. Spectrophotometric Analysis. A UV-1800 spectrophotometer (Shimadzu Scientific Instruments, Kyoto, Japan) was used to record a UV-vis spectrum $(220-700 \mathrm{~nm}$, interval $1 \mathrm{~nm})$ of each sample.

2.2.5. RP-UHPLC-PDA Analysis. Samples were separated on a Thermo Vanquish UHPLC system (Thermo Scientific, Waltham, MA) equipped with a pump, a degasser, an autosampler, and a photodiode array detector (PDA). Samples $(1 \mu \mathrm{L})$ were injected onto a Hypersil GOLD column $(2.1 \times 150 \mathrm{~mm}$, particle size $1.9 \mu \mathrm{m}$; Thermo Scientific). The flow rate used was $400 \mu \mathrm{L} / \mathrm{min}$ at a column temperature of $30^{\circ} \mathrm{C}$. The mobile phases used were $0.1 \%(\mathrm{v} / \mathrm{v}) \mathrm{FA}$ in water (A) and $0.1 \%(\mathrm{v} / \mathrm{v}) \mathrm{FA}$ in $\mathrm{ACN}(\mathrm{B})$. The elution program used was as follows: isocratic at $0 \% \mathrm{~B}$ from 0.00 to $1.09 \mathrm{~min}$, linear gradient to $40 \%$ B from 1.09 to $22.89 \mathrm{~min}$, linear gradient to $100 \%$ from 22.89 to $23.98 \mathrm{~min}$, and isocratic at $100 \% \mathrm{~B}$ from 23.98 to $29.43 \mathrm{~min}$. The eluent was adjusted to its starting composition in $1.09 \mathrm{~min}$, followed by equilibration for $5.48 \mathrm{~min}$. Detection wavelengths for UV-vis were set to the range of $190-680 \mathrm{~nm}$, and data were recorded at $20 \mathrm{~Hz}$.

2.2.6. Electrospray Ionization Ion Trap Mass Spectrometry (ESI$I T-M S^{n}$ ). Mass spectrometric data were acquired using an LTQ Velos Pro linear ion trap mass spectrometer (Thermo Scientific) equipped with a heated ESI probe coupled in-line to the RP-UHPLC system. Nitrogen was used both as sheath gas (47 arbitrary units) and auxiliary gas (11 arbitrary units). Data were collected in negative ionization mode over the $\mathrm{m} / \mathrm{z}$ range $150-1500$ for untargeted analysis and 300-1500 for targeted analysis of higher molecular weight compounds. Data-dependent $\mathrm{MS}^{n}$ analyses were performed by collision-induced dissociation (CID) with a normalized collision energy of $35 \% . \mathrm{MS}^{n}$ fragmentation was performed on the most intense product ion in the $M S^{n-1}$ spectrum. Dynamic exclusion with a repeat count of 3 , repeat duration of $5 \mathrm{~s}$, and an exclusion duration of $5 \mathrm{~s}$ was used to obtain $\mathrm{MS}^{n}$ spectra of multiple different ions present in full MS at the same time. Most settings were optimized by automatic tuning using LTQ Tune Plus 4.2 in Xcalibur 4.2 (Thermo Scientific). The ion-transfer tube temperature was $350{ }^{\circ} \mathrm{C}$, the source heater 
A

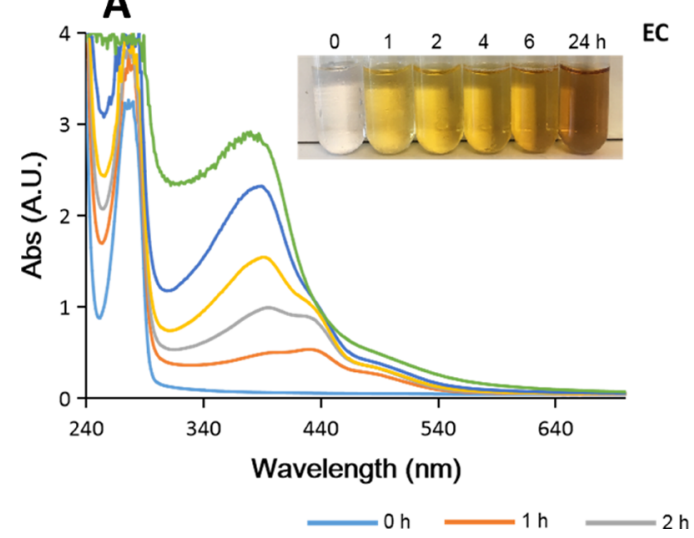

B



Figure 2. UV-vis spectra of the incubated solutions of EC (A) and EGC (B), upon heating at $80^{\circ} \mathrm{C}$ for $0,1,2,4,6$, and 24 h. Insets show pictures of the model systems at the same time intervals.

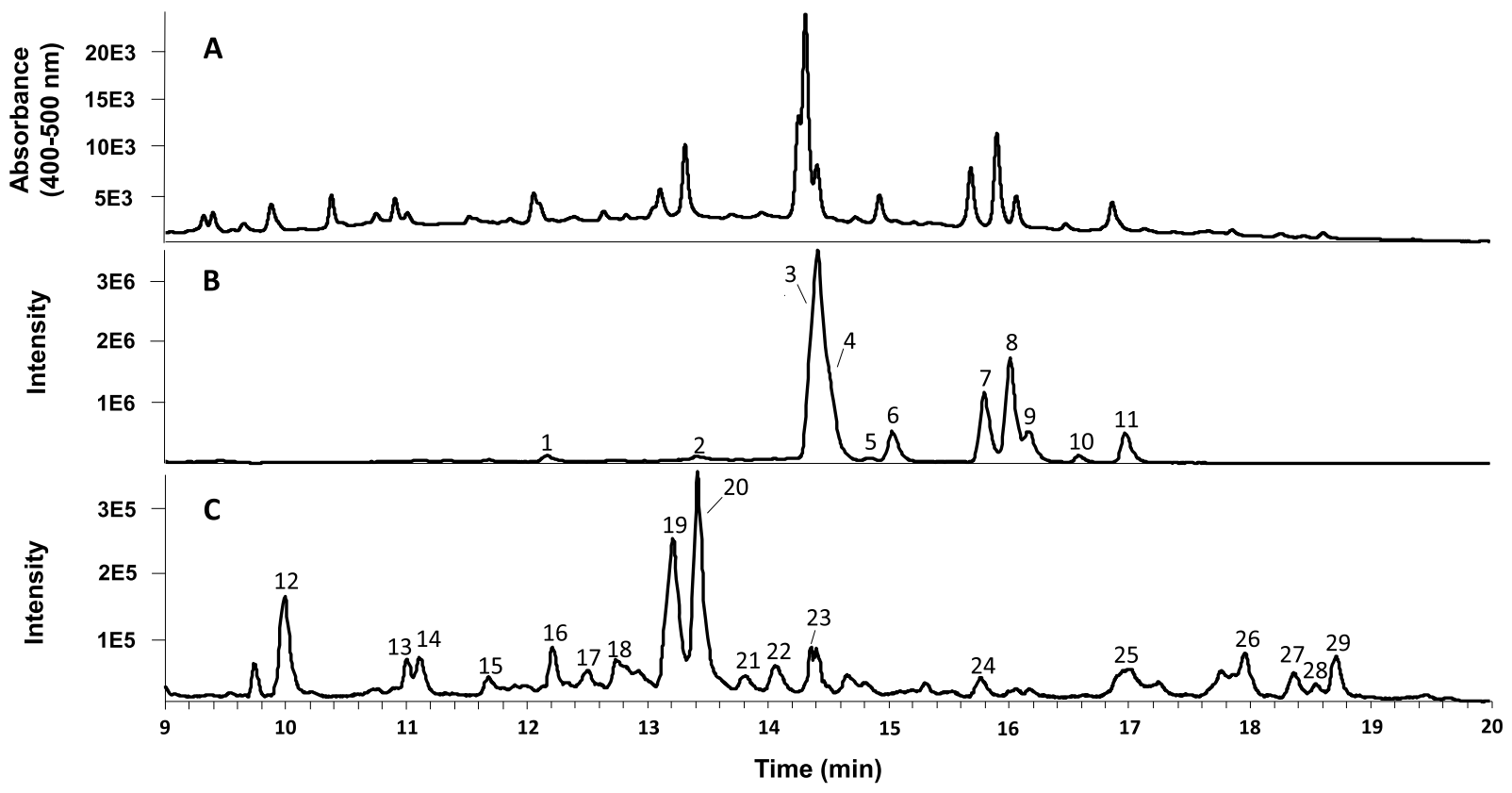

Figure 3. UV-vis chromatogram of absorbance at 400-500 nm (A) and extracted ion chromatograms (negative ionization mode) (B, C) of EC incubated for $6 \mathrm{~h}$. Measured MS range was $\mathrm{m} / z$ 565-596 for detection of dimers, extracted ion $\mathrm{m} / z 575(\mathrm{~B})$; measured MS range was $\mathrm{m} / z$ 855870 for detection of trimers, extracted ions $m / z 861$ and 863 (C). Tentative identification of the numbered peaks is given in Table S1.

temperature was $408{ }^{\circ} \mathrm{C}$, and the source voltage was $4.0 \mathrm{kV}$. Data were processed using Xcalibur 4.2 (Thermo Scientific).

2.2.7. Electrospray lonization Hybrid Quadrupole-Orbitrap Mass Spectrometry (ESI-FTMS). Accurate mass data were acquired using a Thermo Q Exactive Focus Hybrid Quadrupole-Orbitrap Fourier transform mass spectrometer (FTMS) (Thermo Scientific) equipped with a heated ESI probe coupled in-line to the RP-UHPLC system. The mass spectrometer was calibrated in negative and positive ionization modes using Tune software 2.9 (Thermo Scientific) by injection of Pierce negative and positive ion calibration solutions (Thermo Scientific). Nitrogen was used both as sheath gas (47 arbitrary units) and auxiliary gas (11 arbitrary units). The source conditions used in negative ionization mode were as follows: capillary temperature of $254^{\circ} \mathrm{C}$, probe heater temperature of $408{ }^{\circ} \mathrm{C}$, source voltage of $2.5 \mathrm{kV}$, and S-Lens RF level of 50. Full MS data were collected in negative ionization mode over the $\mathrm{m} / z$ range $150-1500$ at 70000 resolution. Higher-energy C-trap dissociation (HCD) fragmentation data were recorded at 70000 resolution. Normalized collision energy (NCE) was varied to obtain spectra that provided maximal structural information. Data were processed using Xcalibur 4.2 (Thermo Scientific).

\section{RESULTS AND DISCUSSION}

3.1. Monitoring Auto-Oxidative Browning. UV-vis spectrophotometry was used to monitor the color formation of the model systems at time points $0,1,2,4,6$, and $24 \mathrm{~h}$. Visual observation of the samples (Figure 2) confirmed that the colorless catechin solutions $(0 \mathrm{~h})$ turned brown after $24 \mathrm{~h}$. Yellowish, brown, or red color is related to absorbance between 400 and $500 \mathrm{~nm}^{14,15}$ An increased intensity of the absorbance of the EC and EGC solutions between 250 and $600 \mathrm{~nm}$, including increased absorbance between 400 and 500 $\mathrm{nm}$, was observed over time (Figure 2). Based on this, the UHPLC-PDA-MS data were screened for UV-vis peaks with absorbance between 400 and $500 \mathrm{~nm}$. These peaks, which corresponded to brown compounds, were matched to the corresponding mass peaks observed in IT-MS or FTMS for further characterization.

3.2. Characterization of Brown Products and Colorless Reaction Intermediates from EC Incubation. For the 

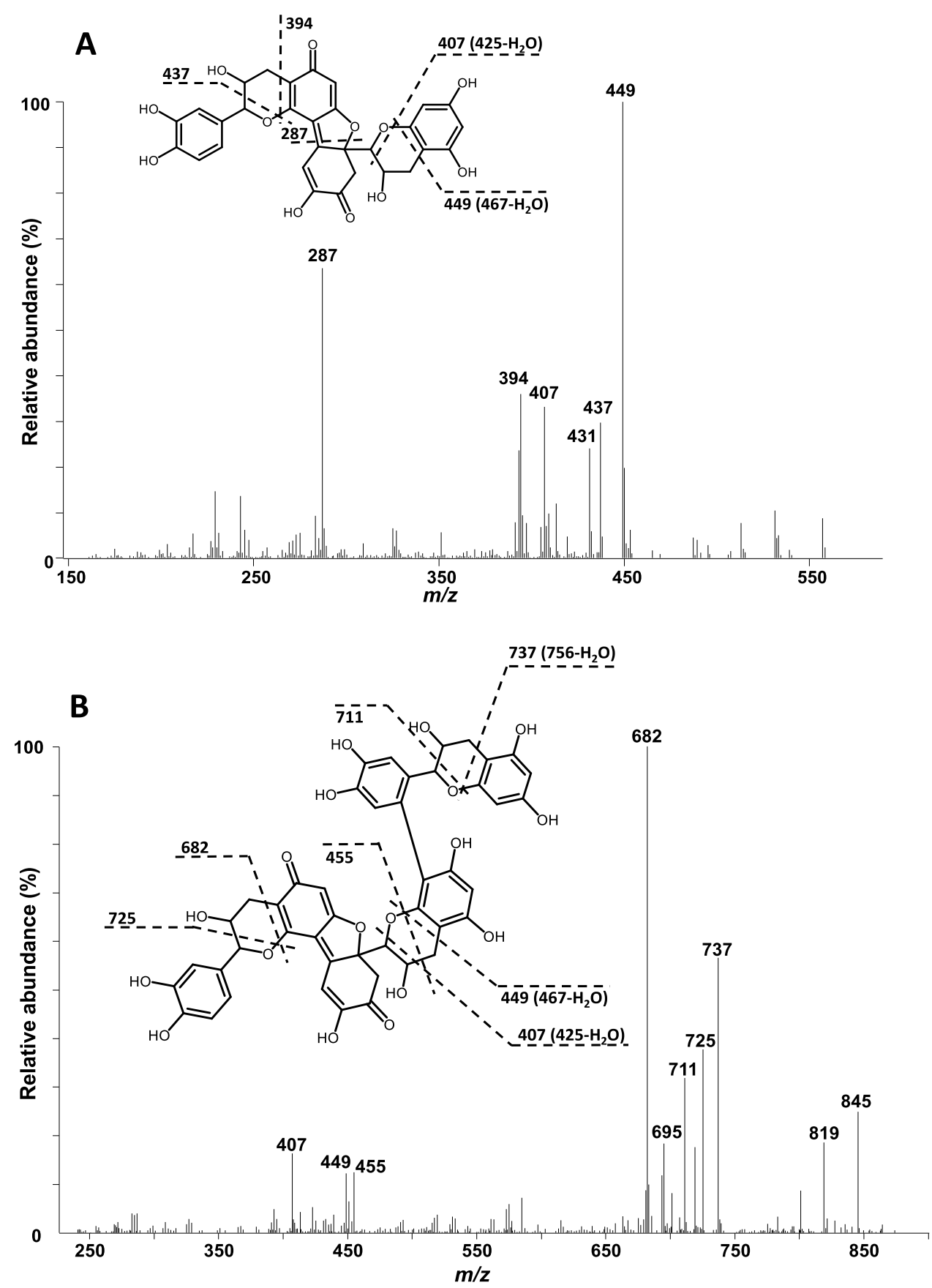

Figure 4. Typical CID MS ${ }^{2}$ fragmentation spectra of $\delta$-type dehydrodicatechin structures with $m / z 575$ (A) and dehydrotricatechins with $m / z$ 863, which possess at least one $\delta$-type interflavanic linkage (B) in negative ionization mode. Dashed lines show the fragmentation pathways.

model incubation of EC, unreacted EC and its epimer catechin (C) are the major compounds found in the UV $280 \mathrm{~nm}$ chromatograms (data not shown) and in the MS base peak chromatograms $(\mathrm{m} / z$ range $150-1500$; Figure S1A). EC and $\mathrm{C}$ are both colorless, i.e., they have no absorbance in the visible light spectrum (380-740 nm). Instead, we focused on compounds with absorbance between 400 and $500 \mathrm{~nm}$, the range in which brown pigments typically absorb light (Figure 3A).

When the EC and C monomers $(m / z 289)$ were excluded from the mass chromatograms (i.e., MS range set to $m / z$ 300$1500)$, peaks with $\mathrm{m} / \mathrm{z}$ values of 575,861 , and 863 were detected as the main oxidation products (Figure S1B) with absorbance between 400 and $500 \mathrm{~nm}$ (Figure 3). This chromatographic peak (UV-vis)-to-peak (full MS) screening indicated that the compounds responsible for browning of the EC model system likely resulted from condensation of EC, yielding dimers or trimers based on the $\mathrm{m} / z$ values detected. Trace amounts of tetramers were detected by MALDI-TOFMS analysis (data not shown); however, due to the low amount of tetramers present in the samples, they were not considered to be of significant influence on the brown color. To gain more insight into the formation of brown dimeric and trimeric oxidation products, MS measurements were performed with narrow mass ranges $(\Delta m / z 15-20)$. From these measurements, extracted ion chromatograms (EIC) were obtained for the specific $\mathrm{m} / \mathrm{z}$ values that correspond to potential catechin dimers and trimers, to relate these 
condensation products to the UV-vis peaks that were detected in the range of $400-500 \mathrm{~nm}$.

The potential dimeric condensation products that were related to brown color were matched to $11 \mathrm{MS}$ peaks with $\mathrm{m} / \mathrm{z}$ 575 (numbered 1-11, Figure 3B) (Figure 3A). These MS peaks showed very similar $\mathrm{MS}^{2}$ fragments (see Table S1). A typical fragmentation spectrum representative of the peaks with $m / z 575$ is shown in Figure 4A. The main $\mathrm{MS}^{2}$ fragment of $\mathrm{m} / z 575$ was $\mathrm{m} / z 449$, resulting from retro-Diels-Alder (RDA) fragmentation of the C-ring in combination with water loss. Besides this, the interflavanic linkage was cleaved resulting in a fragment at $m / z 287$, and additional RDA fragments were observed at $m / z 394,407$, and 437 . The RDA fragmentation of $\mathrm{m} / z 575$ was also confirmed by comparison with the general fragmentation patterns described for flavanols. ${ }^{16-20}$ Following the $\mathrm{MS}^{2}$-based decision tree for catechin oligomers, ${ }^{16}$ if the ratio of the relative intensities of $m / z 449-394$ is larger than 1 , then the compound of $m / z 575$ should be tentatively identified as $\mathrm{EC}-\delta_{\mathrm{AB}}$-EC (i.e., $\delta$-type dehydrodicatechins $\left(\mathrm{DhC}_{2} \mathrm{~s}\right)$ ). The theoretical number of $\delta$-type $\mathrm{DhC}_{2}$ isomers is 18 as predicted by Verloop and co-workers, ${ }^{16}$ which includes positional isomers of the $\mathrm{A}-\mathrm{B}$ ring interflavanic linkage and stereoisomers from $\mathrm{C}$-ring epimerization. These positional isomers or stereoisomers could not be distinguished based on $\mathrm{MS}^{2}$ data.

Besides the brown $\delta$-type $\mathrm{DhC}_{2}$ s, several peaks with $m / z 577$ (Figure S1B), which possessed no absorbance in the range of 400-500 nm, were prominent in the full MS chromatograms. Based on a comparison of their CID fragmentation spectra (Figure S2) with the MS ${ }^{2}$-based decision tree, ${ }^{16}$ these colorless dimers were tentatively identified as $\beta$-type $\mathrm{DhC}_{2}$ s. These $\beta$ type $\mathrm{DhC}_{2}$ s also possess an $\mathrm{A}-\mathrm{B}$ ring interflavanic linkage between two EC subunits. These results suggested that the auto-oxidation process may be a multistep oxidation process, similar to the oxidative cascade during the enzymatic oxidation. ${ }^{17,21}$ The colorless $\beta$-type $\mathrm{DhC}_{2} \mathrm{~s}$ are potential intermediates for the formation of the brown $\delta$-type $\mathrm{DhC}_{2} \mathrm{~s}$.

Besides the formation of EC dimers, the detection of peaks with higher $m / z$ values suggested that oligomers of at least 3 EC subunits were formed. With RP-UHPLC-IT-MS, $18 \mathrm{MS}$ peaks (numbered 12-29, Figure 3C) with $\mathrm{m} / \mathrm{z}$ values of 861 or 863 were found to be relevant to browning. The peaks with $\mathrm{m} / z 863$ were tentatively identified as dehydrotricatechins $\left(\mathrm{DhC}_{3} \mathrm{~s}\right)$ by following the same approach used for $\mathrm{DhC}_{2} \mathrm{~s}$, based on their $\mathrm{MS}^{2}$ fragmentation (data listed in Table S1) and the decision tree. ${ }^{16}$ Based on our annotations, each brown $\mathrm{DhC}_{3}(\mathrm{~m} / z$ 863) possessed two different interflavanic linkages, which included one $\delta$-type linkage and one $\beta$ - or $\varepsilon$-type linkage. This is illustrated with the example of EC- $\delta_{\mathrm{AB}}-\mathrm{EC}-\beta_{\mathrm{AB}}$ EC (Figure 4B). The most abundant MS ${ }^{2}$ signals of $m / z 863$ corresponded to RDA fragments, which is similar to fragmentation of $\delta$-type $\mathrm{DhC}_{2} \mathrm{~s}(\mathrm{~m} / z 575)$ in Figure 4A. The peaks with $m / z 861$ were also tentatively identified as $\mathrm{DhC}_{3} \mathrm{~s}$, with two sets of interflavanic linkages, by the same approach. These peaks possessed more diverse spectral properties, indicating that they could consist of a mixture of different combinations of $\delta$-type and $\gamma$-type linkages (Table S1).

Overall, the auto-oxidative browning products of EC are mainly $\delta$-type dehydrodicatechins and dehydrotricatechins that possess at least one $\delta$-type interflavanic linkage.

3.3. Characterization of Brown Products from EGC Incubation. Similar to our observations for EC, upon incubation of EGC, the most abundant peaks detected by
RP-UHPLC-IT-MS corresponded to EGC and its epimer gallocatechin (GC) (Figure S3). Additionally, theasinensins $(\mathrm{m} / z$ 609) were detected; however, they are colorless and will not be discussed further.

The maximum intensities of the UV-vis absorbance in the range of 400-500 $\mathrm{nm}$ were approximately 15-fold lower for EGC (Figure S3A) than for EC (Figure 4A). Upon matching the main UV-vis peaks $(400-500 \mathrm{~nm})$ to full MS peaks, 13 MS peaks with a mass value of $m / z 551$ (numbered 30-42, Figure S4B) and 2 MS peaks with a mass value of $m / z 399$ (numbered 43-44, Figure S4C) were linked to brown products. The corresponding compounds were tentatively identified by comparison of their spectra with products reported for enzymatic oxidation of EGC. ${ }^{22-24}$

The brown products with $m / z 399$ were tentatively identified as theaflagallins by comparison of their $\mathrm{MS}^{2}$ spectra (Figure S5) to those of previously reported benzotropolone pigments. ${ }^{22}$ Two detected theaflagallin C-ring stereoisomers (epimers) showed similar fragment patterns, such as $\mathrm{m} / z 233$ (loss of C- and A-rings), $m / z 381$ (water loss), $m / z 261$ (RDA fragment on the C-ring), $m / z 273$ (RDA fragment and water loss), and $m / z 355$ (RDA fragment). The elemental composition of $m / z 233.04576\left(\mathrm{C}_{13} \mathrm{H}_{9} \mathrm{O}_{6}\right)$ and $\mathrm{m} / z$ 261.04071 $\left(\mathrm{C}_{12} \mathrm{H}_{9} \mathrm{O}_{5}\right)$ was confirmed by RP-UHPLC-FTMS (Figure S6). To the best of our knowledge, this is the first time that the formation of theaflavin-like compounds as products of auto-oxidation of EGC has been described.

The MS spectra and $\mathrm{MS}^{2}$ fragments of the peaks with $\mathrm{m} / \mathrm{z}$ 551 matched with dihydro-indene-carboxylic acid derivatives of EGC. One of such compounds was previously reported as an enzymatic oxidation product. ${ }^{23}$ These structures feature a carboxyl group on a fused-ring system of a five- and sixmembered ring. However, more peaks were detected than what could be attributed to stereoisomers of the previously reported structure (Table S2), indicating that positional isomers were also formed. Peaks with similar $\mathrm{MS}^{2}$ fragmentation patterns were tentatively assigned as stereoisomers (e.g., epimers), whereas peaks with different $M S^{2}$ fragmentation patterns were assigned as isomers with different positions of the carboxyl group. The main $\mathrm{MS}^{2}$ fragments of $m / z 551$ (Figure S7) were $m / z 533$ (water loss), $m / z 507$ (RDA fragment), $m / z 489$ (RDA fragment and water loss), $m / z 370$ (loss of the A-C ring), and $m / z 383$ (RDA fragment on the C-ring). Additionally, HCD fragmentation in RP-UHPLC-FTMS was used to confirm the molecular formula of the diagnostic fragments used to distinguish the positional isomers. The $\mathrm{MS}^{2}$ fragment of $m / z 232.03812\left(\mathrm{C}_{12} \mathrm{H}_{8} \mathrm{O}_{5}\right.$, Figure S8) indicated that the carboxyl group should be present at the position marked in blue. The $\mathrm{MS}^{2}$ fragment of $\mathrm{m} / z 305.06693$ $\left(\mathrm{C}_{15} \mathrm{H}_{13} \mathrm{O}_{7}\right.$, Figure S9) indicated that the carboxyl group should be present at the position marked in green. Thus, we propose that the main positional isomers are dihydro-indene-7carboxylic acid and dihydro-indene-3-carboxylic acid derivatives of EGC. These compounds are new positional isomers of the previously reported dihydro-indene-4-carboxylic acid derivative of EGC.

Overall, two main types of auto-oxidative browning products of EGC could be identified via the chromatographic peak (UV-vis)-to-peak (full MS) matching strategy, namely, theaflagallins and dihydro-indene-carboxylic acid derivatives of EGC.

3.4. EC and EGC Auto-Oxidative Browning Mechanism. Based on the characterization of brown products 


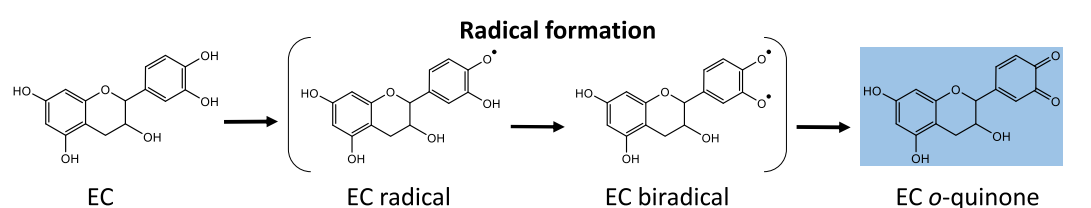

EC radical

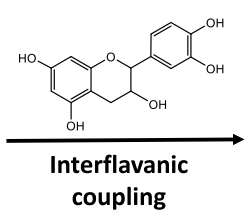

coupling

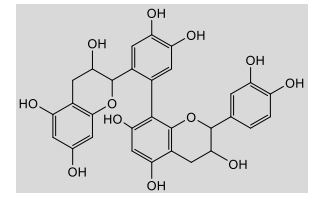

$\beta$-type $\mathrm{DhC}_{2}$

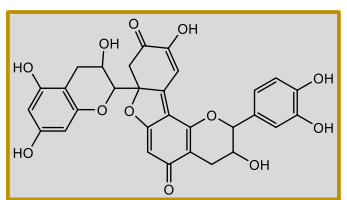

ס-type $\mathrm{DhC}_{2}$

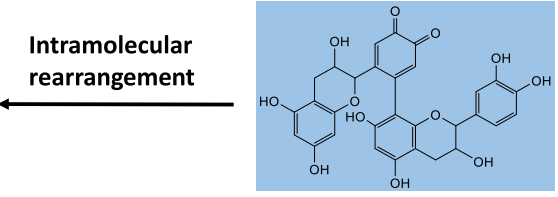

$\beta$-type $\mathrm{DhC}_{2}$ o-quinone<smiles>Oc1cc(O)c2c(c1)OC(c1ccc(O)c(O)c1)C(O)C2</smiles>

EC

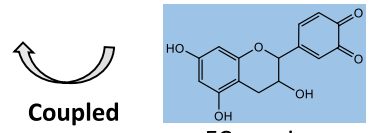

oxidation

EC o-quinone

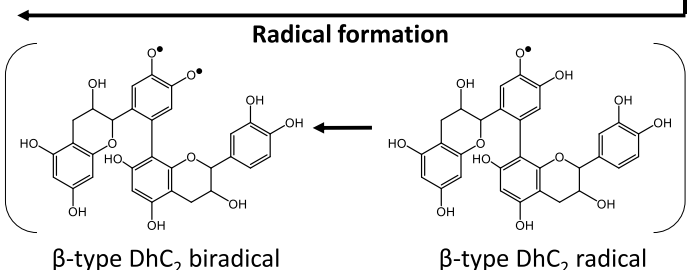

Figure 5. Proposed reaction pathway of EC leading to formation of the main auto-oxidative browning compound, $\delta$-type $\mathrm{DhC}_{2}$ (yellow outline). Gray-shaded compounds represent reaction products detected in the EC model incubation. Blue-shaded compounds represent intermediates detected by trapping with $o$-phenylenediamine. Radical intermediates (in brackets) are proposed based on the literature. ${ }^{26}$

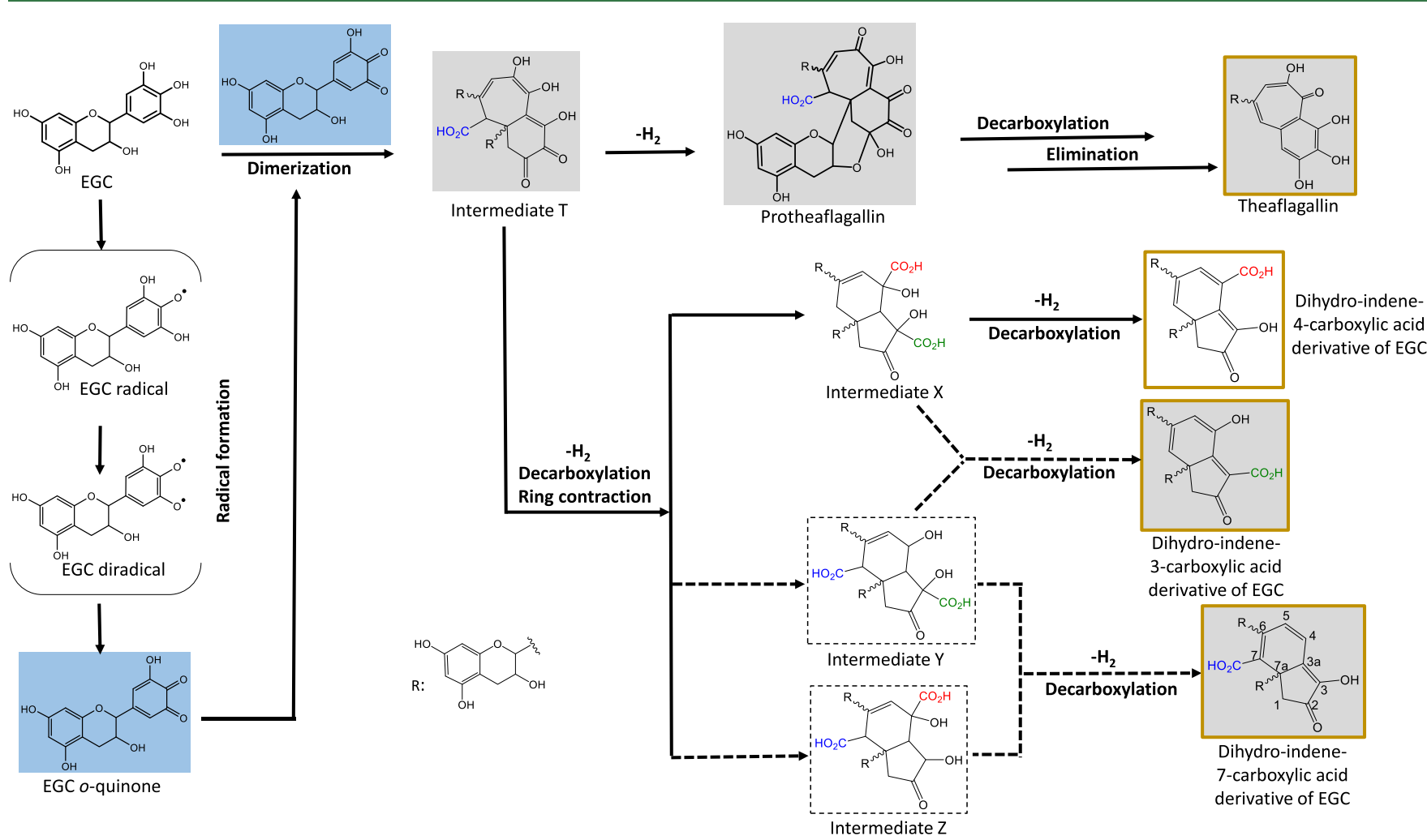

Figure 6. Proposed reaction pathway of EGC leading to auto-oxidative browning compounds (yellow outline). Theaflagallins and dihydro-indene4-carboxylic acid derivative of EGC (carboxyl group in red with gold outline) were previously reported as enzymatic oxidation browning products of EGC. $^{22,23}$ Gray-shaded compounds represent reaction products detected in the EGC model incubation. Blue-shaded compounds represent intermediates detected by trapping with $o$-phenylenediamine. Radical intermediates (in brackets) are proposed based on the literature. ${ }^{26}$ The proposed reaction pathway (dashed arrows) leading to novel dihydro-indene-carboxylic acid derivatives of EGC (carboxyl groups in green or blue) is drawn based on pathways reported for enzymatic oxidation. ${ }^{23}$ The structures of novel intermediates $\mathrm{Y}$ and $\mathrm{Z}$ were proposed based on previously reported intermediate $\mathrm{X}$. 
resulting from EC incubation, $\delta$-type DhCs were identified as the major contributors to browning. The $\delta$-type interflavanic linkage can only be generated through a multistep oxidation reaction, analogous to the oxidative cascade described for enzymatic oxidation. ${ }^{17,21}$ A proposed pathway for nonenzymatic formation of $\delta$-type $\mathrm{DhC}_{2} \mathrm{~s}$ is shown in Figure 5. We hypothesize that the conversion to brown polymers was initiated by two subsequent hydrogen abstractions leading to $o$ quinone formation, with simultaneous reduction of oxygen species. $^{25,26}$ The formation of $o$-quinones upon auto-oxidation in our model system was confirmed by trapping with $o$ phenylenediamine (Figures S10 and S11). The EC $o$-quinone intermediate can participate in subsequent coupling reactions, similar to what is described for the oxidative cascade. ${ }^{27,28}$ The first reaction step is interflavanic oxidative coupling, in which $\mathrm{EC}$ is coupled with an EC $o$-quinone via nucleophilic attack, yielding colorless $\beta$-type $\mathrm{DhC}_{2}$ s with $m / z 577$ (Figure S1B). These compounds are formed through a single $\mathrm{C}-\mathrm{C}$ bond between the A-ring of one $\mathrm{EC}$ subunit and the B-ring of another EC subunit, while simultaneously the EC $o$-quinone subunit is reduced back to an $o$-dihydroxybenzene. ${ }^{17,29}$ The $\beta$ type $\mathrm{DhC}_{2}$ dimers are assumed to serve as intermediates that can undergo either coupled oxidation or radical-initiated oxidation, leading to formation of $\beta$-type $\mathrm{DhC}_{2} o$-quinone (Figure S11). Finally, the $\beta$-type $\mathrm{DhC}_{2} o$-quinone intermediate undergoes intramolecular rearrangement to form $\delta$-type $\mathrm{DhC}_{2} \mathrm{~s}$ $(m / z 575)$ as the stable brown products.

For the EGC incubation, we combined our findings with existing reports from enzymatic oxidation browning products of EGC to propose a reaction pathway explaining the formation of the detected brown products (Figure 6). ${ }^{22,23}$ The auto-oxidation reaction of EGC is also proposed to be initiated by $o$-quinone generation. The formation of EGC $o$ quinones was confirmed by trapping with $o$-phenylenediamine in our model system (Figure S12). The EGC o-quinone can participate in subsequent reactions forming the fused-ring intermediate, $\mathrm{T}{ }^{23} \mathrm{~T}$ is a key interflavanic coupling product for further reactions to produce theaflagallins and dihydro-indenecarboxylic acid derivatives of EGC according to the reported enzymatic reaction pathway. ${ }^{23}$ Most likely, the auto-oxidative formation of the dihydro-indene-7-carboxylic acid and dihydro-indene-3-carboxylic acid derivatives of EGC proceeded via a sequence of decarboxylations and ring contractions. Based on our results, we suggest that the formation of different positional isomers of dihydro-indenecarboxylic acid reactions is influenced by the sequence in which these reactions take place and that this sequence can differ from what was proposed for the dihydro-indene-4carboxylic acid derivative of EGC. We hypothesize that intermediates $\mathrm{Y}$ and $\mathrm{Z}$ will be involved in the formation of these novel dihydro-indene-carboxylic acid derivatives (Figure 6).

3.5. Key Structural Elements of Brown Products Formed upon Auto-Oxidation of EC and EGC. We showed that browning of catechins can occur nonenzymatically in the absence of polyphenol oxidase or peroxidase. The main EC auto-oxidative browning products were identified as $\delta$-type DhCs, which possess an interflavanic double $\mathrm{C}=\mathrm{C}$ bond plus a conjugated carbonyl group $(\mathrm{C}=\mathrm{O}$ bond $)$ on each $\mathrm{EC}$ subunit. These brown products were formed by oxidative coupling via an $o$-quinone mechanism similar to enzymatic oxidation, followed by further oxidation, and subsequent intramolecular rearrangement. Despite the fact that the starting compounds in our aqueous model differed from the complex mixture of catechins present in green tea, we observed brown color formation by structural motifs that were previously reported to be formed during the enzymatic oxidation processes in black tea production. However, the main compounds responsible for the brown color formed upon auto-oxidation were $\delta$-type $\mathrm{DhC}_{2}$ s.

The EGC auto-oxidative browning products were found to be quite diverse. Even though a large number of individual compounds may collectively contribute to browning of EGC, we were able to identify two main types of brown products via our chromatographic peak (UV-vis)-to-peak (full MS) matching strategy. The two main types of brown products are the theaflagallins, which possess a theaflavin-like sevenmembered ring with a carbonyl group, and dihydro-indenecarboxylic acid derivatives of EGC, which include several new positional isomers. These brown compounds possess a fused five- and six-membered ring system with a conjugated carbonyl group and a carboxylic acid moiety.

This study provides the first insight into the mechanisms underlying auto-oxidative browning of catechins. The complex mixtures of coupling products formed upon auto-oxidation of EC and EGC model systems hint at the fact that multicompound systems will pose even greater analytical challenges. Therefore, it will be essential for follow-up studies to study auto-oxidation of galloylated catechins (epigallocatechin gallate and epicatechin gallate) and combinations of catechins, before extending the obtained knowledge toward catechin-rich food products, like RTD green tea. Furthermore, it is expected that additional factors in food products, such as presence of antioxidants, metal ions, and lower $\mathrm{pH}$, will affect autooxidative browning of catechins. ${ }^{30-32}$

Overall, all of the main structures found to contribute to auto-oxidative browning of EC and EGC possessed a combination of an extended conjugated system with fused rings and at least one conjugated carbonyl group.

\section{ASSOCIATED CONTENT}

\section{Supporting Information}

The Supporting Information is available free of charge at https://pubs.acs.org/doi/10.1021/acs.jafc.0c05716.

RP-UHPLC-IT-MS base peak chromatograms of EC and EGC incubation samples in negative ionization mode (Figures S1 and S3); typical CID $\mathrm{MS}^{2}$ fragmentation spectrum of $\beta$-type $\mathrm{DhC}_{2}$, theaflagallin, dihydro-indene-carboxylic acid derivative of EGC, phenazine derivative of epicatechin, phenazine derivative of $\beta$-type $\mathrm{DhC}_{2}$, and phenazine derivative of epigallocatechin (Figures S2, S5, S7, S10, S11, and S12); UV-vis chromatogram and the extracted ion chromatograms in negative ionization mode from $6 \mathrm{~h}$ EGC incubation (Figure S4); high-resolution HCD $\mathrm{MS}^{2}$ fragmentation spectra of theaflagallin, dihydro-indene-7-carboxylic acid derivative of EGC, and dihydro-indene-3-carboxylic acid derivative of EGC in negative ionization mode (Figures S6, S8, and S9); and compounds tentatively identified by RP-UHPLC-IT-MS ${ }^{n}$ in EC and EGC samples after incubation (Tables S1 and S2) (PDF) 


\section{AUTHOR INFORMATION}

\section{Corresponding Authors}

Zhi Lin - Tea Research Institute, Chinese Academy of Agricultural Sciences, Hangzhou, Zhejiang 310008, People's Republic of China; $\odot$ orcid.org/0000-0001-5976-1806; Phone: +86-571-86650617; Email: linzhi@caas.cn Jean-Paul Vincken - Laboratory of Food Chemistry, Wageningen University, 6700 AA Wageningen, The Netherlands; $\odot$ orcid.org/0000-0001-8540-4327; Phone: +31-317-482234; Email: jean-paul.vincken@ wur.nl

\section{Authors}

Junfeng Tan - Tea Research Institute, Chinese Academy of Agricultural Sciences, Hangzhou, Zhejiang 310008, People's Republic of China; Laboratory of Food Chemistry, Wageningen University, 6700 AA Wageningen, The Netherlands

Wouter J. C. de Bruijn - Laboratory of Food Chemistry, Wageningen University, 6700 AA Wageningen, The Netherlands; @ orcid.org/0000-0003-0564-9848

Annemiek van Zadelhoff - Laboratory of Food Chemistry, Wageningen University, 6700 AA Wageningen, The Netherlands; ๑ orcid.org/0000-0001-9416-8979

Complete contact information is available at: https://pubs.acs.org/10.1021/acs.jafc.0c05716

\section{Funding}

The China Scholarship Council (CSC201803250034) partially supported Junfeng Tan's research.

\section{Notes}

The authors declare no competing financial interest.

\section{REFERENCES}

(1) Bradfield, A. E.; Penny, M.; Wright, W. B. The catechins of green tea. J. Acoust. Soc. Am. 1947, 103, 3-5.

(2) Bradfield, A. E.; Bate-Smith, E. C. Chromatographic behaviour and chemical structure II. The tea catechins. Biochim. Biophys. Acta 1950, 4, 441-444.

(3) Stodt, U. W.; Blauth, N.; Niemann, S.; Stark, J.; Engelhardt, U. $\mathrm{H}$.; et al. Investigation of processes in black tea manufacture through model fermentation (oxidation) experiments. J. Agric. Food Chem. 2014, 62, 7854-7861.

(4) Fan, F. Y.; Shi, M.; Nie, Y.; Zhao, Y.; Ye, J. H.; Liang, Y. R. Differential behaviors of tea catechins under thermal processing: Formation of non-enzymatic oligomers. Food Chem. 2016, 196, 347354.

(5) Chen, Z. Y.; Zhu, Q. Y.; Tsang, D.; Huang, Y. Degradation of green tea catechins in tea drinks. J. Agric. Food Chem. 2001, 49, 477482.

(6) Yoshinori, T.; Hiroshi, I.; Hiroaki, H.; Akira, T. Studies on the mechanism of the oxidation of tea leaf catechins. Agric. Biol. Chem. 1964, 28, 64-71.

(7) Davis, A. L.; Lewis, J. R.; Cai, Y.; Powell, C.; Davis, A. P.; Wilkins, J. P. G.; Pudney, P.; Clifford, M. N. A polyphenolic pigment from black tea. Phytochemistry 1997, 46, 1397-1402.

(8) Kuhnert, N.; Clifford, M. N.; Müller, A. Oxidative cascade reactions yielding polyhydroxy-theaflavins and theacitrins in the formation of black tea thearubigins: Evidence by tandem LC-MS. Food Funct. 2010, 1, 180-199.

(9) Matsuo, Y.; Okuda, K.; Morikawa, H.; Oowatashi, R.; Saito, Y.; Tanaka, T. Stereochemistry of the black tea pigments theacitrins A and C. J. Nat. Prod. 2016, 79, 189-195.
(10) Tanaka, T.; Matsuo, Y.; Saito, Y.; Kusano, R. Oxidation mechanism of black tea pigment theaflavin by peroxidase. Tetrahedron Lett. 2015, 56, 5099-5102.

(11) Kim, E. S.; Liang, Y. R.; Jin, J.; Sun, Q. F.; Lu, J. L.; Du, Y. Y.; Lin, C. Impact of heating on chemical compositions of green tea liquor. Food Chem. 2007, 103, 1263-1267.

(12) Li, N.; Taylor, L. S.; Ferruzzi, M. G.; Mauer, L. J. Kinetic study of catechin stability: effects of $\mathrm{pH}$, concentration, and temperature. J. Agric. Food Chem. 2012, 60, 12531-12539.

(13) Bohin, M. C.; Vincken, J.-P.; Van Der Hijden, H. T. W. M.; Gruppen, H. Efficacy of food proteins as carriers for flavonoids. J. Agric. Food Chem. 2012, 60, 4136-4143.

(14) Tilley, R. J. D. Colours Due to Refraction and Dispersion. In Colour and the Optical Properties of Materials, 1st ed.; Tilley, R. J. D., Ed. John Wiley \& Sons, Ltd: UK, 2010; pp 49-90.

(15) Cuthbert, I. D.; del Giorgio, P. Toward a standard method of measuring color in freshwater. Limnol. Oceanogr. 1992, 37, 13191326.

(16) Verloop, A. J.; Gruppen, H.; Vincken, J.-P. Annotation of different dehydrocatechin oligomers by MS/MS and their occurrence in black tea. J. Agric. Food Chem. 2016, 64, 6011-6023.

(17) Guyot, S.; Vercauteren, J.; Cheynier, V. Structural determination of colourless and yellow dimers resulting from (+)-catechin coupling catalysed by grape polyphenoloxidase. Phytochemistry 1996, $42,1279-1288$.

(18) Weinges, K.; Huthwelker, D. Isolierung und konstitutionsbeweis eines 8.6'-verknüpften dehydro-dicatechins (B4). Justus Liebigs Ann. Chem. 1970, 731, 161-170.

(19) Weinges, K.; Mattauch, H. Der chemische konstitutionsbeweis des dehydro-dicatechins A. Chem. -Ztg. 1971, 95, 155-156.

(20) Ma, Y. L.; Li, Q. M.; Van, dH. H.; Claeys, M. Characterization of flavone and flavonol aglycones by collision-induced dissociation tandem mass spectrometry. Rapid Commun. Mass Spectrom. 2015, 11, 1357-1364.

(21) Kuhnert, N.; Drynan, J. W.; Obuchowicz, J.; Clifford, M. N.; Witt, M. Mass spectrometric characterization of black tea thearubigins leading to an oxidative cascade hypothesis for thearubigin formation. Rapid Commun. Mass Spectrom. 2010, 24, 3387-3404.

(22) Matsuo, Y.; Tanaka, T.; Kouno, I. A new mechanism for oxidation of epigallocatechin and production of benzotropolone pigments. Tetrahedron 2006, 62, 4774-4783.

(23) Matsuo, Y.; Hayashi, T.; Saito, Y.; Kouno, I.; Tanaka, T. Structures of enzymatic oxidation products of epigallocatechin. Tetrahedron 2013, 69, 8952-8958.

(24) Drynan, J. W.; Clifford, M. N.; Obuchowicz, J.; Kuhnert, N. The chemistry of low molecular weight black tea polyphenols. Nat. Prod. Rep. 2010, 27, 417-462.

(25) Nakao, M.; Takio, S.; Ono, K. Alkyl peroxyl radical-scavenging activity of catechins. Phytochemistry 1998, 49, 2379-2382.

(26) Waterhouse, A. L.; Laurie, V. F. Oxidation of wine phenolics: A critical evaluation and hypotheses. Am. J. Enol. Vitic. 2006, 57, 306313.

(27) Aniszewski, T.; Lieberei, R.; Gulewicz, K. Research on catecholases, laccases and cresolases in plants. Recent progress and future needs. Acta Biol. Cracov., Ser. Bot. 2008, 1, 7-18.

(28) Yoruk, R.; Marshall, M. R. Physicochemical properties and function of plant polyphenol oxidase: A review. J. Food Biochem. 2003, 27, 361-422.

(29) Sang, S.; Cheng, X.; Stark, R.; Rosen, R.; Yang, C.; Ho, C.-T. Chemical studies on antioxidant mechanism of tea catechins: Analysis of radical reaction products of catechin and epicatechin with 2,2diphenyl-1-picrylhydrazyl. Bioorg. Med. Chem. 2002, 10, 2233-2237.

(30) Komatsu, Y.; Suematsu, S.; Hisanobu, Y.; Saigo, H.; Matsuda, R.; Hara, K. Effects of $\mathrm{pH}$ and temperature on reaction kinetics of catechins in green tea infusion. Biosci. Biotechnol. Biochem. 1993, 57, 907-910.

(31) Bradshaw, M. P.; Prenzler, P. D.; Scollary, G. R. Ascorbic acidinduced browning of $(+)$-catechin in a model wine system. J. Agric. Food Chem. 2001, 49, 934-939. 
(32) Oszmianski, J.; Cheynier, V.; Moutounet, M. Iron-catalyzed oxidation of (+)-catechin in model systems. J. Agric. Food Chem. 1996, $44,1712-1715$. 